\title{
Interdisciplinary Educational Checklist for Allogeneic Stem Cell Transplant Patients
}

\author{
JEAN A. RIDGEWAY, DNP, APN, NP-C, AOCN ${ }^{\circledR}$
}

From University of Chicago Medical Center, Chicago, llinois

Author's disclosures of conflicts of interest are found at the end of this article.

Correspondence to: Jean A. Ridgeway, DNP, APN, NP-C, AOCN® , Department of Medicine, Section of Hematology/Oncology, The University of Chicago Medicine, 5841 S. Maryland Ave., MC 2115, Chicago, IL 60637. E-mail: ridgewayjean@gmail.com

https://doi.org/10.6004/jadpro.2018.9.6.7

(c) 2018 Harborside $^{T M}$
J Adv Pract Oncol 2018:9(6):646-654

\begin{abstract}
Allogeneic hematopoietic stem cell transplant (HSCT) has emerged as a unique treatment modality. Patients who receive an allogeneic HSCT report feeling inadequately equipped to manage their postdischarge plan of care. It is essential that interdisciplinary team members prepare HSCT patients with the education needed in order to deal with the overwhelming care needs involved during and after hospital discharge. Targeted interventions that promote effective, meaningful education and behaviors are needed to guide patients and caregivers through this treatment experience together. Health-care checklists have produced dramatic, sustained gains in patient safety and quality of care. Checklists provide an ideal way to comply with standards of evidencebased care and promote good communication among interdisciplinary team members. The main purpose of this project was to investigate the use of an educational checklist for allogeneic stem cell transplant patients by interdisciplinary team members. Provider use of the educational checklist was evaluated at discharge assessing completion of checklist items.
\end{abstract}

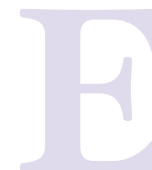

very year in the United States, thousands of adults are diagnosed with lifethreatening hematologic malignancies such as leukemia and lymphoma. A successful treatment option for some of these individuals is an allogeneic hematopoietic stem cell transplant (HSCT). According to the Center for International Bone and Marrow Transplant Research, in 2015 (D'Souza \& Zhu, 2016), 8,351 allogeneic HSCTs were performed for patients over the age of 20 in the United States for various hematologic malignancies, and over 20,000 were performed worldwide (D'Souza \& Zhu, 2016). The number of HSCT recipients is expected to grow by two or three times by 2020 as advances in transplant techniques and supportive care practices lead to progressive improvements in survival for recipients (Majhail et al., 2012).

Patients who have had an HSCT have distinctive health care and ed- 
ucational needs (Rice \& Bailey, 2009). Trembly (2013) reported that nearly half of HSCT patients and caregivers felt inadequately equipped to manage their postdischarge care plan. It is of utmost importance to prepare HSCT patients and their caregivers to be properly educated in order to deal with the overwhelming tasks involved during and after hospital discharge. This will enable the HSCT patient and their caregiver to safely manage health needs and medical complexities at home.

\section{BACKGROUND AND SIGNIFICANCE}

Hematopoietic stem cell transplant has become the standard of care for many patients with diseases of the hematopoietic system, such as hematopoietic malignancies (Cooke, Grant, \& Gemmill, 2012). Hematopoietic stem cell transplant recipients are perhaps the most medically complex population among patients with cancer because of the number of complications, increased mortality, long trajectory of rehabilitation, immune function complexity, and the patient and procedure intensity (Cooke et al., 2012; Rice \& Bailey, 2009). The patient and the assigned caregiver must demonstrate an understanding of the transplant process, be aware of conditions or symptoms that herald potential emergencies, and be active participants in the patient's care for the transplant process to be successful (Cooke et al., 2012; Rice \& Bailey, 2009).

Patients with all types of cancer typically report that they want to know all the information about their disease and its treatment; however, they are often overwhelmed by the amount of information provided by clinicians and media (Epstein \& Street, 2007). Holloway (1996) reported that patients' knowledge and information at the time of hospital discharge can be minimal and, in many informational areas of need, patients felt they were not given essential specifics. Strategies to ensure that patients are informed are important. Readmission rates of $51 \%$ have been reported with HSCT patients; however, adequate educational preparation throughout the care cycle and especially at hospital discharge has the potential to affect readmissions (Grant, Cooke, Bhatia, \& Forman, 2005).

Many unique health-care professionals, including nurses, advanced practice providers, physicians, pharmacists, and social workers provide information to patients in all stages of their treat- ment. This points to an interdisciplinary approach for successful patient education. Drinka and Clark (2000) put forth a widely used definition of "interdisciplinary" as individuals working together in a group to solve problems too complex to be addressed by one discipline or multiple disciplines acting in sequence. From the initial diagnosis to the end of life, patients with cancer receive optimal care when their health-care professionals not only collaborate but strive to learn from each other (Knoop, Wujcik, \& Wujcik, 2017).

Health-care checklists have produced dramatic, sustained gains in patient safety and quality of care by hardwiring best practices, fostering accountability, and improving team communication (Gawande, 2009). The educational needs of allogeneic HSCT patients exemplify the type of clinical problem that is ideally suited to checklist use: it is a complex, dynamic, high-acuity process, carried out by the interdisciplinary team (IDT) amidst multiple competing priorities, often in the setting of time pressure and suboptimal communication (Hales \& Pronovost, 2006). In their sentinel paper, Pronovost and colleagues (2008) presented a project improving patient safety in intensive care units (ICUs) as a collaborative, evidence-based project using a checklist. The checklist was one component of a comprehensive program to alter the culture of the ICUs, which also included empowering nurses to stop procedures if checklist guidelines were not followed. This study modeled how to achieve results when implementing a practice change using a checklist: recruit a team, keep them focused on goals, create an alliance with administration to secure resources, shift power relations, open channels of communication, and give feedback to improve results. Pronovost and colleagues (2008) concluded that using checklists requires focused effort that is properly informed by a scientifically grounded understanding of how organizations and people work, based on theory and evidence.

Checklists are used commonly to ensure that all aspects of an activity are provided and nothing is overlooked (Hales \& Pronovost, 2006). Checklists can also provide consistency by ensuring that each IDT member providing education is presenting the same content to each patient. Checklists can ensure completeness and consistency and provide a means of documenting that education was conducted, 
in addition to what was reviewed and discussed (Mueller \& Glennon, 2007). In recent years, checklists have emerged and are known to have gained considerable support, showing both a decrease in complications and 30-day mortality (Pronovost et al., 2006). Good checklists are the tool, not the goal, in the scheme of improving outcomes for patients.

\section{PURPOSE}

The purpose of the project was to develop and use an interdisciplinary educational checklist for allogeneic stem cell transplant patients (see Appendix A). The University of Chicago Medical Center has been performing HSCTs since October 1985. Over 500 stem cell transplant procedures occurred in the years between 2008 and 2016 (BMT InfoNet, 2017). This project was submitted and approved by the University of Chicago Institutional Review Board.

Posttransplant education is complex, involving multiple medications and significant lifestyle changes (Cooke et al., 2012). Complex medical regimens and multiple medications can make it more likely that patients will misunderstand or forget instructions, or become confused about dosing; interdisciplinary teaching with a checklist can hopefully minimize these complexities. Checklists provide an ideal way to comply with standards of evidence-based care and promote good communication among caregivers (Ashbrook, Mourad \& Sehgal, 2013).

Based on the literature, no matter how expert one may be in any field, a well-designed checklist can improve outcomes (Gawande, 2009; Soong et al., 2013; Trembly, 2013). Gawande (2009) reports that it is far from obvious that something as simple as a checklist could be of substantial help. Checklists remind individuals of the necessary steps and make them explicit. Soong and colleagues (2013) describe a structured approach to discharge planning, starting from admission and proceeding through discharge, using a standardized interdisciplinary checklist beginning on the day of admission. Checklists provide an ideal way to comply with standards of evidence-based care and promote communication among patients, caregivers, and providers.

\section{DESCRIPTION}

This project was a prospective single-institution pilot study that developed and implemented an interdisciplinary educational checklist for allogeneic
HSCT (see Appendix A) into clinical practice. The primary subjects of this project were the members of the allogeneic stem cell transplant program IDT: attending physicians, registered nurses, transplant research nurses, advanced practice providers, social workers, pharmacists, pastoral care, nutritionists, physical therapists, occupational therapists, recreation therapists, and oncology fellows. The primary subjects' use of the checklist as a group was evaluated during this project.

The secondary subjects for this project were the adult allogeneic HSCT patients (age > 20) undergoing a first transplant procedure (Table 1). Patients' eligibility and exclusion criteria (Table 2) were met in order to be consented and participate in the project.

\section{IMPLEMENTATION}

A brief overview of the steps required for this project evaluating the primary subjects (IDT members) is as follows. The project was presented at the weekly leukemia, lymphoma, and stem cell transplant conferences prior to its initiation to inform IDT members of the purpose and methods. A self-paced learning module designed to educate IDT members on the purpose of the project and the checklist was emailed following in-service presentations. The learning module showcased how to use the checklist when educating allogeneic HSCT patients and how to properly document education completed on the checklist. Multiple in-service presentations at various times of the day were conducted over a 2 -week period. These presentations provided in-person education to the inpatient nursing, pharmacy, dietary, and physical therapy staff to reinforce the checklist and answer questions from staff regarding the project. Key points, a self-paced learning module, key articles, and a sample checklist were made available via a binder on the transplant inpatient unit for IDT members to review at their convenience. In addition, key stakeholders were informed about upcoming in-services via personal communication and email blasts.

First, IDT members were consented and asked to sign the signature log. Following IDT consent, they were requested to document all education/ teaching conducted with consented HSCT patients via the checklist document. Charge nurses 


\begin{tabular}{|c|c|}
\hline & $\mathbf{N}$ \\
\hline \multicolumn{2}{|l|}{ Age } \\
\hline $23-70$ years old $($ median $=50)$ & 20 \\
\hline \multicolumn{2}{|l|}{ Gender } \\
\hline Male & 14 \\
\hline Female & 6 \\
\hline \multicolumn{2}{|l|}{ Race } \\
\hline White & 12 \\
\hline African American & 6 \\
\hline Asian or Pacific Islander & 2 \\
\hline \multicolumn{2}{|l|}{ Marital status } \\
\hline Married & 15 \\
\hline Divorced & 3 \\
\hline Single & 2 \\
\hline \multicolumn{2}{|l|}{ Diagnosis } \\
\hline AML & 11 \\
\hline $\mathrm{NHL}$ & 5 \\
\hline MDS & 2 \\
\hline ALL & 1 \\
\hline AA & 1 \\
\hline \multicolumn{2}{|c|}{$\begin{array}{l}\text { Note. } A M L=\text { acute myelogenous leukemia; } \\
\text { NHL = non-Hodgkin lymphoma; MDS = myelodysplastic } \\
\text { syndrome; ALL = acute lymphoblastic leukemia; } \\
\text { AA = aplastic anemia. }\end{array}$} \\
\hline
\end{tabular}

communicated to their staff the names of participating patients and a discussion of checklist use was included in each daily nursing "huddle" meeting. Eligible allogeneic HSCT patients were identified by the project leader and contacted via a face-to-face meeting. The project leader entered each patient's room, provided an introduction, and explained the purpose of the project. Patients were asked if they were interested in participating in the project. Patients who agreed were consented and given a project folder. Each folder contained patient instructions, a thank-you letter for participating, and a copy of the signed study consent. The project leader reviewed each item in the folder with the patient and answered all of the patient's questions. The patient was advised that he/she needed to have the checklist in their room at all times as an inpatient, and would surrender the checklist to the project leader or a member of the advanced practice staff upon discharge from the inpatient unit. The inpatient nursing staff was notified of the patient's participation in the check-

\section{Table 2. Inclusion and Exclusion Criteria} for Patients

Eligibility criteria

- $\geq 20$ years of age

- Able to read and write English

- Planning to undergo or undergoing an allogeneic HSCT in the next 4 weeks

- Alert and orientated to person, place, and time

- Physically present at University of Chicago Medical Center

- Diagnosed with any hematologic disease

Exclusion criteria

- $\geq$ second allogeneic HSCT

- Only orientated to person

- Unwilling to participate

Note. HSCT $=$ hematopoietic stem cell transplant.

list study, as were members of the IDT either via email or face-to-face discussion.

This was a multiphase project that was conducted over a 6-month period. There were 82 IDT members (Figure 1) and 22 patients consented, with 20 completed checklists at the time of data analysis. There were no deaths or withdrawals during this project. Two patients did not proceed to transplant due to disease progression. While patients were hospitalized for conditioning chemotherapy and stem cell infusion and recovery, the IDT used the checklist. Percentage completion of the checklist was measured at the time of discharge. Consented IDT members were asked to document all education/teaching conducted with consented HSCT patients via the checklist.

\section{RESULTS}

Average percentage of completion of the checklist at discharge was 76\% (Figure 2). Percentage of completion of each checklist was measured for all 25 items on the checklist. In order for a content item to be considered "completed," a checkmark and at least one IDT member's initials were required to complete the item content.

Out of all the documentation completed, physicians accounted for $5 \%$ use, while those with a nursing education background (registered nurses and advanced practice nurses) accounted for $95 \%$. No other IDT members were documented completing items on the 20 checklists.

Checklist data were evaluated according to five domain areas: inpatient information, self-care education, monitoring and response to complica- 


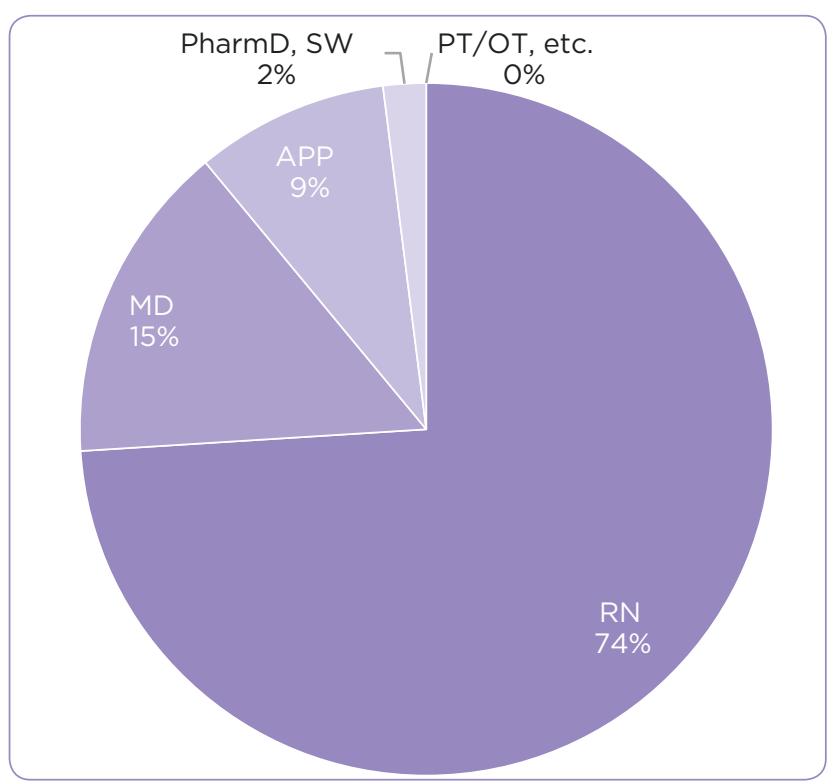

Figure 1. Providers consented $(\mathrm{N}=82)$.

$\mathrm{RN}=$ registered nurse; $\mathrm{MD}=$ medical doctor; APP = advanced practice provider;

PharmD = Doctor of Pharmacy;

$\mathrm{SW}=$ social worker; $\mathrm{PT}$ = physical therapist;

OT = occupational therapist.

tions, exercise and nutrition, and adapting to posttransplant status (Figure 3). The domain of monitoring and response to complications was the most documented (93\%), while the domain of inpatient information was the least documented (67\%).

\section{DISCUSSION}

Overall compliance from IDT members was lower than expected. Registered nurses and advanced practice providers were the most frequent users of the checklist. No physical or occupational therapists, pharmacists, or social workers documented on the checklist, although they are integral members of the HSCT interdisciplinary team. Physicians gave verbal acceptance of the checklist and rounded on all patients daily; however, they rarely documented on the checklist. Lack of use does not imply IDT education was not completed. However, it was not recorded via the checklist. Providers remarked that they did not complete the checklist because it was a paper tool unable to be included in electronic medical record (EMR) documentation, and therefore required extra time and attention to complete. Interdisciplinary team members were unfamiliar with the routine use of the checklist with an 8-week time frame of data collection.

The roles of the IDT members allowing them to function largely independently to provide care for the same patient may provide one explanation

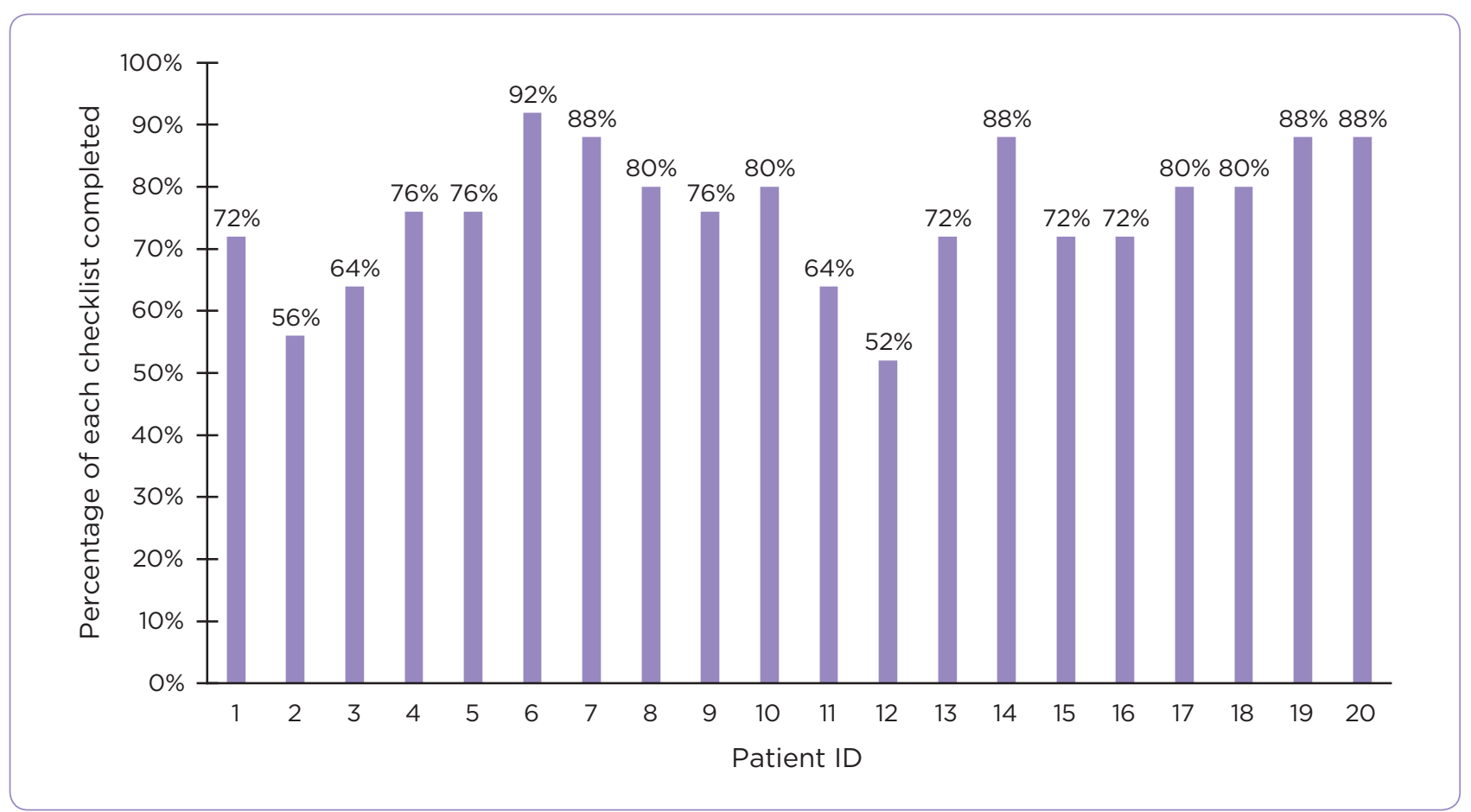

Figure 2. 25-item interdisciplinary team checklist results. This table shows the total percentage completed of each patient's checklist. The highest was $92 \%$ and the lowest was $52 \%$. The average was $76 \%$. 


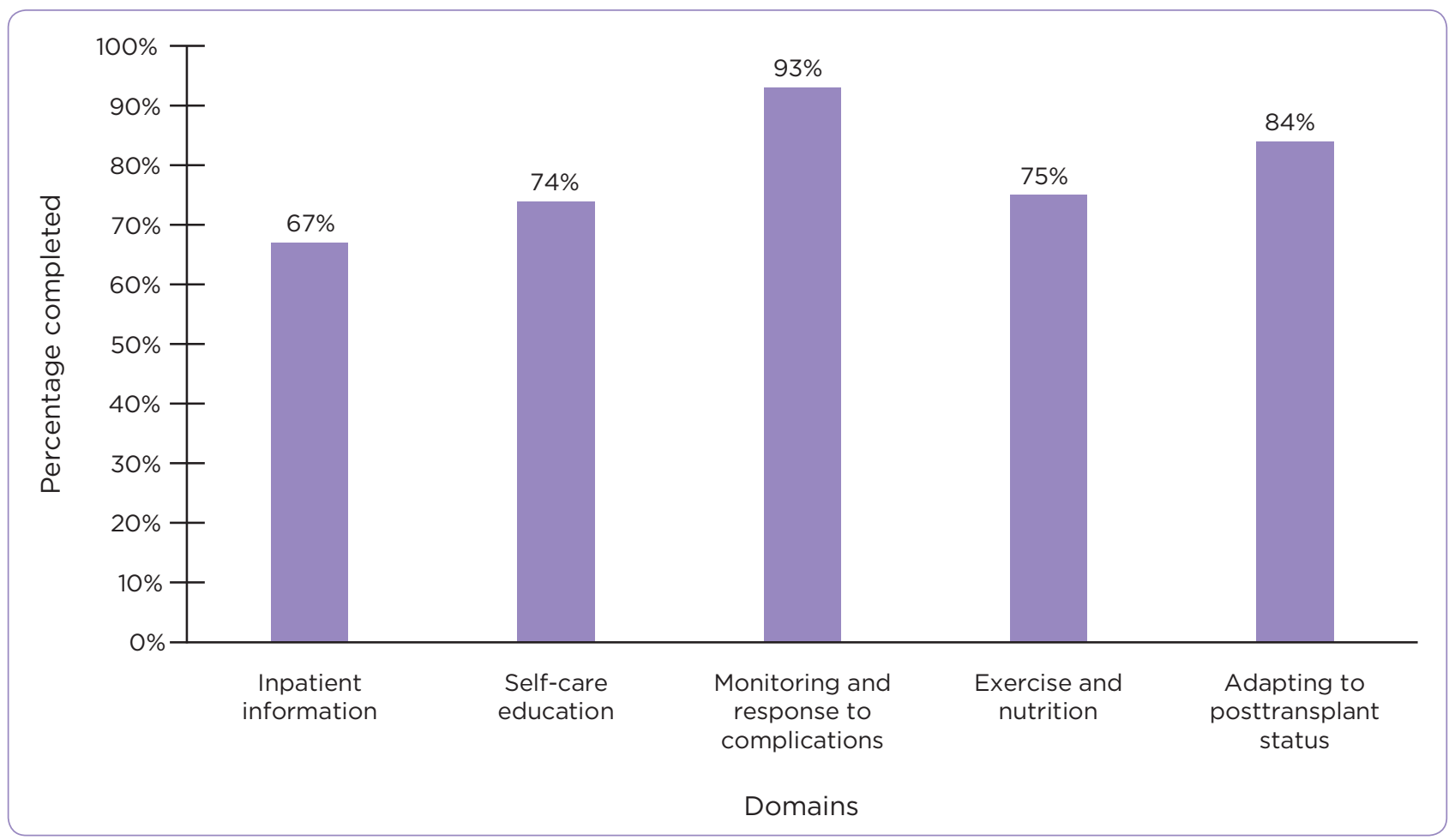

Figure 3. Percentage of domain completion. Each domain was addressed at least $50 \%$ of the time, with the domain of monitoring and response to complications being the domain most often addressed.

of the low use of the checklist. For example, physical therapy is ordered by the advanced practice provider and MD IDT members and provided by a physical therapist, who is also part of the IDT. However, there are limited mechanisms for communication and coordination between physical therapy and the other IDT members, and this may account for the lack of use of the checklist. However, this does not represent fragmented care. Other IDT members, such as registered nurses and advanced practice providers, deliver care in a highly interdependent way to achieve mutually agreedon care objectives. Vogel \& Hall (2016) report that such team members typically have a shared team identity and team objectives, a shared mental model of how team members interact to achieve team objectives, and systems for information sharing and team communication. This may represent the consistent use of the IDT checklist by registered nurses and advanced practice providers.

Although the sample size was small, trends of completing education and taking credit for education via documentation can be seen. Nursing as a discipline incorporates education for the patient at every level. Nurses continued to provide personal- ized education to HSCT patients even though they verbalized their perception that they did not have "enough time" to do so. An interdisciplinary checklist can only be as interdisciplinary as those who participate and document on the checklist. In this case, although the checklist was designed to include all IDT members, the results show that registered nurses, advanced practice providers, and physicians were the sole representatives on the checklist.

The results demonstrated that the domains addressed by the highest percentage of IDT members were monitoring and response to complications (93\%) and adapting to posttransplant status (84\%). The percentages of IDT members addressing the domains of inpatient information (67\%) and self-care education (74\%) were the lowest documented (Figure 3). This may reveal the lack of expertise of the IDT, which consisted primarily of registered nurses and advanced practice providers, in instructing the patients, and exposes a lack of familiarity with the information for these domain areas. Yet, at least $50 \%$ of the time, IDT members discussed each domain.

The most valued comments came from the patients. Patients remarked that they would encour- 
age IDT members to use the checklist daily and were frustrated by the lack of concern and use of the checklist by IDT members, even though education occurred.

Study limitations included a small sample size $(n=20)$. The small sample size reflected a limited 8-week time of data collection. Sample size and time of data collection may have skewed the results of this project. The subjects were a convenience sample of adult patients, and the results may differ in another location.

\section{Barriers}

The barrier of resistance and low compliance of using the checklist by the IDT was anticipated. The project leader was aware that use of the checklist depended on the practicality of the checklist and perceived usefulness of the idea. Without documentation on the checklist that education was completed, it could not be stated with certainty that education was done. Upon informal discussion with many of the IDT members, there was a repeated theme of "not having enough time" or simply "forgetting" to complete the checklist. The barrier of adopting the checklist for completing and documenting patient education decreased over the course of the project.

An unexpected barrier of the IDT members was their lack of expertise with the HSCT content material that needed to be discussed with patients. A group of key stakeholders who could address this deficiency were identified and education was conducted to address the problem. The project leader developed content and conducted formal team meetings to generate objectives and assigned responsibilities to specific team members to enhance the knowledge of various IDT members. Training and education play an important role in overcoming resistance in the adoption of a new initiative. The project leader was confident that conducting training with the IDT would help to ensure the success of this project.

\section{IMPLICATIONS FOR PRACTICE}

The use of the checklist in this project was viewed from a feasibility perspective rather than that of effectiveness or statistical significance. The project was intended to implement an evidence-based project into a health-care setting. There exists inherit care complexity with HSCT patients, yet all patients require information on their treatment, side effects, and self-care needs. The IDT also inserts a level of communication complexity. However, this communication complexity can be bridged by collaboration in the truest sense of the word, and thereby share best practices across the IDT and transform patient care. Interdisciplinary team members can assume other disciplines are completing education and documentation; however, if documentation is not completed, patient education cannot be confirmed.

Having an interdisciplinary educational checklist has the potential to identify problems early and set in motion interventions on an individual basis and prescribe a comprehensive interdisciplinary plan of care designed to meet the patients' needs. Interdisciplinary team members found difficultly when using a checklist despite the recognition of a positive tool. Interdisciplinary team members each play a unique role in the care of SCT patients and have the opportunity to address the educational needs of patients and caregivers at each treatment phase on the trajectory of transplant care. Lessons learned from this project include the need to consider what is necessary to engage the IDT in patient education and what is necessary to sustain this engagement.

\section{CONCLUSION}

The way in which patient education is documented by the IDT can be a platform for interprofessional collaboration. It can create a forum of sharing medical information, as well as provide an opportunity to teach others to promote optimal patient care. Creating an IDT educational checklist as a documentation template in our EMR is the next step for this project. The development of an IDT tool is underway amidst the complexity of providing an easy way for all IDT members to use it. Having an interdisciplinary educational checklist has the potential to identify problems early and set in motion interventions on an individual basis and prescribe a comprehensive interdisciplinary plan of care designed to meet the patients' needs.

Organizational change, although positive, can be difficult to embrace in a short amount of time. "Documentation fatigue" or "documentation burden" is a real phenomenon and needs to 
be addressed for IDT members. This project made a contribution to understanding the adoption of a practice change and the complexities of introducing a new tool to the practice environment.

\section{Acknowledgment}

I would like to thank my principal investigator and faculty mentor, Dr. Andrew Artz, for his assistance with this project, as well as the RN/APN staff of the University of Chicago for participating in this project.

\section{Disclosure}

The author has no conflicts of interest to disclose.

\section{References}

Ashbrook, L., Mourad, M., \& Sehgal, N. (2013). Communicating discharge instructions to patients: A survey of nurse, intern, and hospitalist practices. Journal of Hospital Medicine, 8(1), 36-41. https://doi.org/10.1002/jhm.1986

BMT InfoNet. (2017). University of Chicago Medical Center/ BMT InfoNet. Retrieved from https://www.bmtinfonet. org/transplantcenter/university-chicago-medical-center

Cooke, L., Grant, M., \& Gemmill, R. (2012). Discharge needs of allogeneic transplantation recipients. Clinical Journal of Oncology Nursing Online Exclusive, 16(4), E142-E149. https://doi.org/10.1188/12.CJON.E142-E149

D'Souza, A., \& Zhu, X. (2016). Current uses and outcomes of hematopoietic cell transplantation (HCT): Center for International Blood and Marrow Transplant Research summary slides. Retrieved from http://www.cibmtr.org

Drinka, T. J. K., \& Clark, P. G. (2000). Healthcare teamwork: Interdisciplinary practice and teaching. Westport, CT: Greenwood Publishing Group.

Epstein, R. M., \& Street, R. L. (2007). Patient-centered communication in cancer care: Promoting healing and reducing suffering. Bethesda, MD: National Cancer Institute.

Gawande, A. (2009). The checklist manifesto: How to get things right. New York, NY: Metropolitan Books.

Grant, M., Cooke, L, Bhatia, S., \& Forman, S. J. (2005). Discharge and unscheduled readmissions of adult patients undergoing hematopoietic stem cell transplantation: Implications for developing nursing interventions. Oncology Nursing Forum, 32(1), E1-E8. https://doi.org/10.1188/05.ONF.E1-E8
Hales, B. M., \& Pronovost, P. J. (2006). The checklist-a tool for error management and performance improvement. Journal of Critical Care, 21(3), 231-235. https://doi. org/10.1016/j.jcrc.2006.06.002

Holloway, A. (1996). Patient knowledge and information concerning medication on discharge from hospital. Journal of Advanced Nursing, 24(6), 1169-1174. https://doi. org/10.1111/j.1365-2648.1996.tb01022.x

Knoop, T., Wujcik D., and Wujcik, K. (2017). Emerging models of interprofessional collaboration in cancer care. Seminars in Oncology Nursing, 33(4), 459-463. https://doi. org/10.1016/j.soncn.2017.08.009

Majhail, N. S., Murphy, E. A., Denzen, E. M., Ferguson, S. S., Anasetti, C., Bracey, A.,...Snyder, E. L. (2012). The National Marrow Donor Program's symposium hematopoietic cell transplantation in 2020: A health care resource and infrastructure assessment. Biology of Blood Marrow Transplantation, 18(2), 172-182. https://doi.org/10.1016/j. bbmt.2011.10.004

Mueller, P. S., \& Glennon, C. A. (2007). A nurse-developed prechemotherapy educational checklist. Clinical Journal of Oncology Nursing, 11(5), 715-719. https://doi. org/10.1188/07.CJON.715-719

Pronovost, P., Needham, D., Berenholtz, S., Sinopoli, D., Chu, H., Cosgrove, S.,...Goeschel, C. (2006). An intervention to decrease catheter-related bloodstream infections in the ICU. New England Journal of Medicine, 355, 2725-2732. https://doi.org/10.1056/NEJMoa061115

Pronovost, P. J., Berenholtz, S. M., Goeschel, C., Thom, I., Watson, S. R., Holzmueller, C. G....Sexton, J. B. (2008). Improving patient safety in intensive care units in Michigan. Journal of Critical Care, 23(2), 207-221. https://doi. org/10.1016/j.jcrc.2007.09.002

Rice, R. D., \& Bailey, G. (2009). Management issues in hematopoietic stem cell transplantation. Seminars in Oncology Nursing, 25(2), 151-158. https://doi.org/10.1016/j. soncn.2009.03.009

Soong, C., Daub, S., Lee, J., Majewski, C., Musing, E., Nord, P.,...Bell, C. M. (2013). Development of a checklist of safe discharge practices for hospital patients. Journal of Hospital Medicine, 8(8), 444-449. https://doi.org/10.1002/ jhm.2032

Trembly, A. (2013). Can a preflight checklist make hospital discharge safer? Nursing Management, 44(1), 8-11. https://doi.org/10.1097/01.NUMA.0000424024.13787.4b

Vogel, A. L., \& Hall, K. L. (2016). Creating the conditions for implementing team principles in cancer care. Journal of Oncology Practice, 12(11), 964-969. https://doi. org/10.1200/JOP.2016.018218 


\section{Appendix A. University of Chicago Medical Center Stem Cell Transplant Education Checklist}

Patient ID:

\section{Educational content}

1. Orientation to inpatient setting: provider's daily schedule, provider rounding, etc.

2. Overview of transplant trajectory

3. Visitor policy

4. Chemotherapy schedule

5. Initiate case manager assessment

6. Assess and educate on ADLs and mobility

7. Physical therapy assessment

8. Occupational/recreational assessment

9. Advance directive needs

10. Chemotherapy side effects

11. Initiate/reinforce venous access teaching

12. Assess nutrition status teaching and needs

13. Diet and food-drug interactions

14. Transplant medications side effects

15. Identify barriers to discharge

16. Diabetic teaching (as needed)

17. Home preparation

18. Fatigue conservation

19. What symptoms to report to your provider

20. How to contact your provider

21. Outpatient routine/follow-up appointments

22. Sexuality

23. Home exercise and walking plan

24. Infection precautions

25. Home medication teaching

Note. Please comment on content and reference any handouts, etc. in comment section. Questions? Call Jean Ridgeway. $\mathrm{MD}=$ medical doctor; $\mathrm{APP}=$ advanced practice provider; $\mathrm{RN}=$ registered nurse;

PharmD = Doctor of Pharmacy; SW = social worker; PT = physical therapist; OT = occupational therapist;

$A D L=$ activities of daily living.

$\begin{array}{cc}\text { MDs } & \text { APPs } \\ \text { (initials/ } & \text { (initials/ } \\ \text { date) } & \text { date) }\end{array}$

RNs

(initials/

SW (initials/

date)

PT/OT, etc.

(date)

date)

Comments
PharmD, 